\title{
BMJ Open Neurocognitive impairment after neonatal sepsis: protocol for a systematic review and meta-analysis
}

\author{
Jen Heng Pek (D) , ${ }^{1}$ Bei Jun Yap, ${ }^{2}$ Ming Ying Gan, ${ }^{2}$ Shu Ting Tammie Seethor, ${ }^{2}$ \\ Rachel Greenberg, ${ }^{3}$ Christoph Paul Vincent Hornik, ${ }^{4}$ Bobby Tan, ${ }^{5}$ Jan Hau Lee, ${ }^{6}$ \\ Shu-Ling Chong (D) ${ }^{5}$
}

To cite: Pek JH, Yap BJ, Gan MY, et al. Neurocognitive impairment after neonatal sepsis: protocol for a systematic review and meta-analysis. BMJ Open 2020;10:e038816. doi:10.1136/ bmjopen-2020-038816

- Prepublication history and additional material for this paper are available online. To view these files, please visit the journal online (http://dx.doi. org/10.1136/bmjopen-2020038816).

Received 25 March 2020 Revised 11 May 2020 Accepted 18 May 2020
Check for updates

(C) Author(s) (or their employer(s)) 2020. Re-use permitted under CC BY-NC. No commercial re-use. See rights and permissions. Published by BMJ.

For numbered affiliations see end of article.

Correspondence to Dr Shu-Ling Chong; chong.shu-ling@kkh.com.sg

\section{ABSTRACT}

Introduction The effect of neonatal sepsis on the developing brain is not well documented. We aim to perform evidence synthesis to determine the outcome of neurodevelopmental impairment and intellectual disability among survivors of neonatal sepsis. The data gathered will inform on the long-term neurocognitive outcomes of neonates with sepsis and the measures used to document their developmental disability.

Methods and analysis We will perform a search based on the following parameters: neonates and infants less than 90 days old diagnosed with sepsis who had neurocognitive outcomes or measures of developmental disability reported. We will search PubMed, Cochrane Central, Embase and Web of Science for articles in English language published between January 2010 and December 2019. Clinical trials and observational studies will be included. Two independent reviewers will screen studies for eligibility. Data extraction will then be performed using a standardised form. The quality of evidence and risk of bias will be assessed using Cochrane Collaboration's tool and Risk of Bias in Non-randomised Studies of Intervention (ROBINS-I). The results will be synthesised qualitatively and pooled for meta-analysis.

Ethics and dissemination No formal ethical approval is required as there is no collection of primary data. This systematic review and meta-analysis will be disseminated through conference meetings and peer-reviewed publications.

PROSPERO registration number Registration submitted CRD42020164334

\section{INTRODUCTION}

Brain development, during which neuronal connections are established and strengthened, is particularly crucial between the late gestational and early neonatal phase. ${ }^{1-3}$ Insults to the developing brain during this period can negatively impact cerebral networking and control, leading to poor mental and psychomotor development, cerebral palsy, visual and auditory impairment, as well as intellectual disability later in life. ${ }^{45}$ In the neonatal period, these insults include prematurity, low birth weight, seizures, hyaline

\section{Strengths and limitations of this study}

- This review will synthesise and update the association between neonatal sepsis and neurocognitive outcomes of neurodevelopmental impairment and intellectual disability over the past decade.

- Subgroup analysis stratified by clinical predictors and geographical locations will be performed to provide further insight about neurocognitive impairment.

- This protocol adheres to Preferred Reporting Items for Systematic Reviews and Meta-Analyses guidelines.

- Heterogeneity across studies may exist due to differences in patient populations, settings and studies performed over a decade.

- The inclusion of only studies published in English may lead to a loss of data published in other languages.

membrane disease, hyperbilirubinemia of the newborn or kernicterus and sepsis. ${ }^{6}$ Among these, neurocognitive outcomes following neonatal sepsis are not well documented for the following reasons: lack of consensus in definition of neonatal sepsis, confounding due to the presence of other neonatal conditions with sepsis, wide variation in measures used to assess and document neurocognitive outcomes, as well as need for long-term follow-up.

The link between neonatal sepsis and adverse neurocognitive outcomes has been postulated to be a multifactorial process involving production of proinflammatory cytokines, hypoxic ischaemic encephalopathy due to hypotension and impaired autoregulation of cerebral blood flow, stimulation of microglia causing excitotoxicity, as well as free radical damage by reactive oxygen and nitrogen species. ${ }^{7-14}$ All these lead to injury in the cerebral white matter, particularly periventricular leukomalacia, 
and increase the vulnerability of the brain to subsequent injuries. ${ }^{15-17}$

Common measures of neurocognitive outcomes include neurodevelopmental impairment and intellectual disability. ${ }^{18-22}$ Neurodevelopmental impairment includes the following: cognitive delay based on standardised cognitive tests (eg, Mental Development Index of Bayley Scales of Infant and Toddler Development), hearing deficits or loss requiring amplification, visual impairment and moderate to severe cerebral palsy defined as a score of two or more on the Gross Motor Function Classification System. ${ }^{23}$ Intellectual disability is a commonly used measure and is defined as a disorder which begins before the age of 18 , characterised by limitations in adaptive behaviour which affect participation in everyday life and at least one of the three domains: conceptual, social and practical, as well as limitations in intellectual functioning which affect general mental capacity and is indicated by an IQ that is 2 SDs below the mean. ${ }^{24} 25$

Both the United Nations and WHO have emphasised the importance of improved health in this vulnerable population. ${ }^{2627}$ As mortality in the under-5 falls, there is an impetus to understand the long-term effect on survivors of common childhood diseases. ${ }^{28}{ }^{29}$ Specific to neonatal sepsis, the knowledge of neurocognitive outcomes will guide future researchers in their understanding and measurement of vital patient-related outcomes.

Current systematic review and/or meta-analyses have shown that neonatal sepsis was associated with an increased risk of adverse neurocognitive outcomes. ${ }^{18-22}$ However, they are limited to very low birth weight infants, ${ }^{18}$ those less than 28 days old, ${ }^{19}$ infection secondary to group B Streptococcus, ${ }^{20}$ those with healthcare-associated infection ${ }^{21}$ and more general neonatal populations (including, but not specific to, sepsis). ${ }^{22}$ Studies also mainly focused on severe neurocognitive delay and did not describe the full range of intellectual disability. ${ }^{20-22}$ Therefore, we intend to perform a large-scale global study across gestation, regardless of pathogen, that will inform us on all severities of neurocognitive delay following neonatal sepsis.

In the systematic review, we aim to appraise and summarise the association between neonatal sepsis and neurocognitive outcomes of neurodevelopmental impairment and intellectual disability. In the meta-analysis, pooled estimates, along with the trends over time, of neurodevelopmental impairment and intellectual disability following neonatal sepsis will be reported.

\section{Methodology}

This review will be conducted using the Preferred Reporting Items for Systematic Reviews and Meta-Analyses 2009 guidelines. ${ }^{30}$

\section{Eligibility criteria}

A study will be included if the study's population is less than 90 days old, regardless of gestation; if the study's population has neonatal sepsis based on the definition or diagnostic criteria as determined by the study authors, regardless of causative organism; and if the study's population has reported neurocognitive outcomes of neurodevelopmental impairment, including mental or psychomotor impairment, visual or auditory impairment and cerebral palsy, as well as intellectual disability. There is no limit to the time frame for which the outcomes are measured. All randomised controlled trials, casecontrol studies, cohort studies and cross-sectional studies published in the English language between January 2010 and December 2019 will be included. We have chosen to limit the study to a 10-year systematic review and metaanalysis because the landscape on epidemiology and case definitions in neonatal sepsis is evolving and our aim is to provide an update to the current literature. ${ }^{31-34}$

Studies with sample size less than 50 are excluded due to publication biases, reporting biases and smallstudy effects that could cause extreme point estimations. Studies focusing solely on necrotising enterocolitis, respiratory distress syndrome, leukaemia and other malignancies without sepsis will also be excluded as the target population is a neonatal population with sepsis, no individual specific conditions mentioned. Furthermore, in these conditions, the course of illness, the use of medications and, therefore, the implication on subsequent neurocognitive deficits are different from a general sepsis population.

All studies included in the study will be assessed for the quality of research using the Quality Assessment Tool for Quantitative Studies, which is developed by the Effective Public Health Practice Project. ${ }^{35}$

\section{Information sources}

The following electronic databases will be covered in the literature search: PubMed, Cochrane Central, Excerpta Medica (Embase) and Web of Science. The following electronic registries for trial protocols will be searched to ensure that there are no completed or ongoing studies which intend to perform a similar neurocognitive outcome study on all neonates with sepsis: PROSPERO, ClinicalTrials.gov, International Standard Randomised Controlled Trial Number registry, WHO International Clinical Trials Registry Platform and European Union Clinical Trials Register.

\section{Search strategy}

The search strategy, developed in consultation with research librarians experienced in systematic reviews and meta-analyses, will include all publications from January 2010 to December 2019. Medical Subject Headings $(\mathrm{MeSH})$ are used for PubMed and Cochrane Central. Emtree terms are used for Embase. Topic terms are used for Web of Science. The terms will be appropriately exploded with inclusion of their synonyms in the title, abstract and keyword searches.

Online supplementary appendices 1-4 show the full search strategy. Strategic search terms include: Population-neonate, newborn, infant and baby; Exposuresepsis, septicaemia, septic shock, pyaemia, endotoxaemia 
and blood poisoning; and Outcome-morbidity, developmental disability, intellectual disability, neurodevelopmental disorders, learning disorder, motor skills disorder, vision disorder, hearing loss and cerebral palsy.

Reference lists of relevant systematic reviews will be screened for primary studies within the publication period from January 2010 to December 2019 to look for potentially missed studies from our search strategy.

\section{Study records}

The review of articles will be performed using Covidence V.1357.0, Melbourne, VIC, Australia. Duplicate studies will be removed and the remaining studies will be screened for eligibility by two independent reviewers. The resolution of any conflicts will be done by a third independent reviewer or by discussion. The reasons for exclusion of any article will be documented. A full text screening will then be carried out.

\section{Data items}

Data extraction will be performed using a standardised form capturing the following data fields: study title, author(s), publication year, geographic origin, study design, enrolment period, sample size, source definitions of neonatal sepsis, classification of neonatal sepsis as early or late, severity of neonatal sepsis, demographics (eg, gestational age, postnatal age, sex and birth weight), comorbidities, source of infection, causative organisms (ie, bacterial, fungal, parasitic or viral), communityacquired or hospital-acquired infection, blood markers (eg, white blood cell count, absolute neutrophil count, C-reactive protein, procalcitonin and lactate), neurodevelopmental outcomes of mental or psychomotor impairment, visual or auditory impairment and cerebral palsy, as well as intellectual disability. We will also include details of any early intervention programmes performed post sepsis in each study. The standardised form will be pilot tested for 20 studies to ensure that data is accurately and consistently captured. Qualitative data will be used for systematic review and quantitative data will be used for meta-analysis.

\section{Outcomes and prioritisation}

The primary outcome of our study is to determine the impact of neonatal sepsis on outcomes of neurodevelopmental impairment and intellectual disability in neonates and infants less than 90 days old. The prior defined outcomes under neurodevelopmental impairment are mental impairment ${ }^{36}$ (measured using the Bayley Scales of Infant and Toddler Development (III): Mental Development Index ${ }^{37}$ ), psychomotor impairment ${ }^{36}$ (measured using the Bayley Scales of Infant and Toddler Development (III): Psychomotor Development Index ${ }^{37}$ ), cerebral palsy, ${ }^{36}$ visual impairment ${ }^{36}$ and auditory impairment. ${ }^{36}$ We will also study cognitive impairment defined as intellectual disability ${ }^{38}$ (measured using the Stanford-Binet Intelligence Scales, ${ }^{39}$ the Wechsler Intelligence Scale ${ }^{40}$ and the Wechsler Preschool and Primary Scale of Intelligence ${ }^{41}$ ).
In the quantitative analysis, the data on these outcomes will be extracted for meta-analysis to calculate effect sizes longitudinally across time periods. The outcomes will be compared across the following subgroups: central nervous system (vs those not involving the central nervous system), gestation age, early (vs late) sepsis, community (vs acquired) infection and the gross national income status where the research was performed. ${ }^{42}$ All studies will be reviewed systematically and the definition of these outcomes used in the study will be described.

\section{Data synthesis}

Each individual study will be assessed for selection bias, performance bias, detection bias, attrition bias and reporting bias by assigning a rating of low, high or unclear risk of bias. Cochrane Collaboration's tool and ROBINS-I will be used to assess for risk of bias for randomised and non-randomised studies, respectively. ${ }^{43}$ The overall strength of the evidence provided by the study will also be evaluated. This information about the included studies will be described and illustrated in a risk of bias graph.

The overall effect will be presented in forest plots. Effect sizes will be calculated for each individual study. These will be determined by a random-effects approachDerSimonian and Laird method, which employs inverse variance. $^{44}$

To address the missing data, original authors will be contacted via email. Available data will be verified by corroborating with data in the original article. If data remain unavailable after two attempts made 1 month apart, we will first perform the analysis excluding these data, followed by a sensitivity analysis assuming that the missing data had negative outcomes. These studies with missing data will also be included in the systematic review, where a table for the findings of each study and a narrative based on synthesis of this information will be presented.

\section{Software to be used}

The software Stata V.16.0 (College Station, Texas, USA) will be used for this meta-analysis.

\section{Meta-bias}

Funnel plot will be used to assess publication bias, systematic difference between higher and lower precision studies, as well as any false effects due to poor methodology of the individual studies.

\section{Patient and public involvement}

This research will be performed without patient and public involvement as this is not an interventional study and does not involve patient enrolment. Patient and public will not be invited to comment on the study design and will not be consulted to develop patient relevant outcomes or interpret the results.

\section{DISCUSSION}

With mortality rates from neonatal sepsis decreasing over time, focus has shifted to caring for the survivors. ${ }^{45}$ Sepsis 
causes significant disruption to cerebral networking in the neonatal period and is detrimental to brain development. Consequentially, neurocognitive impairment of motor, cognitive, language, learning and behavioural skills can occur and will negatively impact a child's performance and integration into school, and eventually into society as an adult. ${ }^{46}{ }^{47}$ Therefore, this systematic review and metaanalysis on neurocognitive outcomes following neonatal sepsis will provide valuable knowledge on the measurable disabilities of these children. In doing so, we can set the direction for future efforts to better study neurocognitive outcomes in this vulnerable population.

The current challenge is that neurocognitive impairment following neonatal sepsis is not well evaluated or understood. Better knowledge on the incidence and impact of this specific problem will enable discretionary resource allocation, especially among groups that are at a high risk for long-term neurocognitive impairments. Interventions can be instituted to improve neurocognitive impairment by generating sensory inputs, such as tactile, proprioceptive, vestibular, visual and auditory sensations to promote brain development. ${ }^{48}$ These interventions need to be started early during the widow of active brain development in order for the stimuli to be effective. ${ }^{40}$ One example is the Newborn Individualised Developmental Care and Assessment Program which trains health professionals to individualise care for the neonate based on his or her behaviour. ${ }^{51}$ Other interventions include motor development, physiotherapy, interaction parenting, social support and upbringing plan. ${ }^{52}$ These interventions have a positive influence on neurocognitive and motor outcomes, which persist later on in life. $^{53}$

It is imperative that we recognise the burden of neonatal sepsis among survivors. They deserve better opportunistic and timely intervention, with particular attention paid to high risk groups. Therein lies the importance of this systematic review and meta-analysis. By synthesising existing literature based on both population and hospital-based studies, we will be able to contribute to a more complete knowledge base about the incidence and impact of neonatal sepsis on neurocognitive impairment, thus facilitating the focus and implementation of intervention programmes, as well as future research to improve care and outcomes for this high-risk population.

\section{Limitations}

The definition and domains of neurocognitive impairment, assessment tools, reference standards and validation for use in the study's context as well as duration of follow-up may vary among studies. This may potentially result in a significant heterogeneity which precludes aggregation of some data. We will document these variations in the studies as a quality measure.

Similarly, there is a lack of consensus-based definitions for neonatal sepsis. ${ }^{34}$ We will address this variation by matching the sepsis definition used in the studies to that of Goldstein et al, which is a systematic inflammatory response syndrome (SIRS) in the presence of or as a result of suspected or proven infection. ${ }^{54}$ This definition is chosen as age-specific reference ranges for vital signs and laboratory variables of SIRS in the neonatal population are clearly stated for referencing.

Next, studies may be missed if they are not indexed under MeSH, Emtree and Topic terms used in our search strategy for the various search engines. Furthermore, the databases may not be exhaustive and only studies published in English language will be included. Inclusion of different study designs in our systematic review and meta-analysis will also result in a variable quality of evidence. Nevertheless, we will assess the risk of bias using Cochrane Collaboration's tool and ROBINS-I for randomised and non-randomised studies, respectively.

Finally, neurocognitive impairment in this age group may be heavily confounded by clinical, social and environmental factors. In particular, we will not be able to account for the quality of care provided outside the medical management of sepsis, in each setting.

This systematic review and meta-analysis of neonatal sepsis and the outcomes of neurocognitive impairment will provide insight to the incidence and impact on long-term healthcare burden of neonatal sepsis among survivors. Ultimately, the knowledge gained will facilitate future research in the identification of high-risk groups and allow for resource planning in this vulnerable population.

\section{Author affiliations}

1Department of Emergency Medicine, Sengkang General Hospital, Singapore

${ }^{2}$ Yong Loo Lin School of Medicine, National University of Singapore, Singapore

${ }^{3}$ Department of Pediatrics, Duke University School of Medicine, Durham, North Carolina, USA

${ }^{4}$ Division of Quantitative Sciences, Department of Pediatrics, Duke University School of Medicine, Durham, North Carolina, USA

${ }^{5}$ Department of Emergency Medicine, KK Women's and Children's Hospital, Singapore

${ }^{6}$ Children's Intensive Care Unit, KK Women's and Children's Hospital, Duke-NUS Medical School, Singapore

Contributors JHP designed the protocol, as well as drafted and revised the paper. BJY, MYG and STTS designed the protocol and data collection tool, as well as revised the paper. $\mathrm{RG}$ and $\mathrm{CH}$ developed the statistical analysis plan and revised the paper. BT and JHL designed the protocol and data collection tool, as well as revised the paper. S-LC initiated the project, designed the protocol and data collection tool, as well as revised the paper.

Funding This work is funded by National Medical Research Council (Grant Number: CSSSP19may-0006).

Competing interests None declared.

Patient consent for publication Not required.

Provenance and peer review Not commissioned; externally peer reviewed.

Open access This is an open access article distributed in accordance with the Creative Commons Attribution Non Commercial (CC BY-NC 4.0) license, which permits others to distribute, remix, adapt, build upon this work non-commercially, and license their derivative works on different terms, provided the original work is properly cited, appropriate credit is given, any changes made indicated, and the use is non-commercial. See: http://creativecommons.org/licenses/by-nc/4.0/.

\section{ORCID iDs}

Jen Heng Pek http://orcid.org/0000-0002-8356-7410

Shu-Ling Chong http://orcid.org/0000-0003-4647-0019 


\section{REFERENCES}

1 Ikonomidou C, Bosch F, Miksa M, et al. Blockade of NMDA receptors and apoptotic neurodegeneration in the developing brain. Science 1999;283:70-4.

2 Poo MM. Neurotrophins as synaptic modulators. Nat Rev Neurosci 2001;2:24-32.

3 Dobbing J, Sands J. Quantitative growth and development of human brain. Arch Dis Child 1973;48:757-67.

4 Tau GZ, Peterson BS. Normal development of brain circuits. Neuropsychopharmacology 2010;35:147-68.

5 Adams-Chapman I. Insults to the developing brain and impact on neurodevelopmental outcome. J Commun Disord 2009;42:256-62.

6 Soleimani F, Zaheri F, Abdi F. Long-Term neurodevelopmental outcomes after preterm birth. Iran Red Crescent Med J 2014;16:e17965

7 Silveira RC, Procianoy RS, Dill JC, et al. Periventricular leukomalacia in very low birth weight preterm neonates with high risk for neonatal sepsis. J Pediatr 2008;84:211-6.

8 Graham EM, Holcroft CJ, Rai KK, et al. Neonatal cerebral white matter injury in preterm infants is associated with culture positive infections and only rarely with metabolic acidosis. Am J Obstet Gynecol 2004;191:1305-10.

9 Miller SP, Ferriero DM, Leonard C, et al. Early brain injury in premature newborns detected with magnetic resonance imaging is associated with adverse early neurodevelopmental outcome. $J$ Pediatr 2005;147:609-16.

10 Khwaja O, Volpe JJ. Pathogenesis of cerebral white matter injury of prematurity. Arch Dis Child Fetal Neonatal Ed 2008;93:F153-61.

11 Eklind S, Hagberg H, Wang X, et al. Effect of lipopolysaccharide on global gene expression in the immature rat brain. Pediatr Res 2006;60:161-8.

12 Hagberg $\mathrm{H}$, Mallard $\mathrm{C}$. Effect of inflammation on central nervous system development and vulnerability. Curr Opin Neurol 2005;18:117-23.

13 Lehnardt S, Lachance C, Patrizi S, et al. The Toll-like receptor TLR4 is necessary for lipopolysaccharide-induced oligodendrocyte injury in the CNS. J Neurosci 2002;22:2478-86.

14 Fleer A, Krediet TG. Innate immunity: Toll-like receptors and some more. A brief history, basic organization and relevance for the human newborn. Neonatology 2007;92:145-57.

15 Hoffmann O, Braun JS, Becker D, et al. TIr2 mediates neuroinflammation and neuronal damage. J Immunol 2007;178:6476-81.

16 Volpe JJ. Postnatal sepsis, necrotizing entercolitis, and the critical role of systemic inflammation in white matter injury in premature infants. J Pediatr 2008;153:160-3.

17 Wang X, Rousset $\mathrm{Cl}$, Hagberg $\mathrm{H}$, et al. Lipopolysaccharide-Induced inflammation and perinatal brain injury. Semin Fetal Neonatal Med 2006;11:343-53.

18 Alshaikh B, Yusuf K, Sauve R. Neurodevelopmental outcomes of very low birth weight infants with neonatal sepsis: systematic review and meta-analysis. J Perinatol 2013;33:558-64.

19 Bakhuizen SE, de Haan TR, Teune MJ, et al. Meta-Analysis shows that infants who have suffered neonatal sepsis face an increased risk of mortality and severe complications. Acta Paediatr 2014; $103: 1211-8$.

20 Kohli-Lynch M, Russell NJ, Seale AC, et al. Neurodevelopmental impairment in children after group B streptococcal disease worldwide: systematic review and meta-analyses. Clin Infect Dis 2017:65:S190-9.

21 Haller S, Deindl P, Cassini A, et al. Neurological sequelae of healthcare-associated sepsis in very-low-birthweight infants: umbrella review and evidence-based outcome tree. Euro Surveill 2016;21:30143.

22 Mwaniki MK, Atieno M, Lawn JE, et al. Long-Term neurodevelopmental outcomes after intrauterine and neonatal insults: a systematic review. Lancet 2012;379:445-52.

23 Wilson-Costello DPost TW, ed. Long-Term neurodevelopmental outcome of preterm infants: epidemiology and risk factors, 2020.

24 Pivalizza PPost TW, ed. Intellectual disability in children: definition, diagnosis, and assessment of needs, 2020.

25 American Association on Intellectual and Developmental Disabilities. Definition of intellectual disability. Available: https://www.aaidd.org/ intellectual-disability/definition [Accessed 29 Apr 2020].

26 United Nations. Sustainable development goals: goal 3. Available: http://www.un.org/sustainabledevelopment/health/\# 6604b7b1968a53ff9 [Accessed 13 Oct 2019].

27 World Health Organization. Global strategy for women's, children's and adolescents' health 2016-2030. Geneva, Switzerland: WHO, 2015.

28 Norheim OF, Jha P, Admasu K, et al. Avoiding 40\% of the premature deaths in each country, 2010-30: review of national mortality trends to help quantify the UN sustainable development goal for health. Lancet 2015;385:239-52.

29 Countdown to 2030 Collaboration. Countdown to 2030: tracking progress towards universal coverage for reproductive, maternal, newborn, and child health. Lancet 2018:391:1538-48.

30 Liberati A, Altman DG, Tetzlaff J, et al. The PRISMA statement for reporting systematic reviews and meta-analyses of studies that evaluate healthcare interventions: explanation and elaboration. BMJ 2009;339:b2700.

31 Bizzarro MJ, Raskind C, Baltimore RS, et al. Seventy-Five years of neonatal sepsis at Yale: 1928-2003. Pediatrics 2005;116:595-602.

32 Bizzarro MJ, Shabanova V, Baltimore RS, et al. Neonatal sepsis 2004-2013: the rise and fall of coagulase-negative staphylococci. $J$ Pediatr 2015;166:1193-9.

33 Wynn JL, sepsis Dneonatal. Defining neonatal sepsis. Curr Opin Pediatr 2016;28:135-40.

34 Wynn JL, Wong HR, Shanley TP, et al. Time for a neonatalspecific consensus definition for sepsis. Pediatr Crit Care Med 2014;15:523-8

35 Effective Public Healthcare Panacea Project.. Quality assessment tool for quantitative studies.. Available: https://www.ephpp.ca/ quality-assessment-tool-for-quantitative-studies [Accessed May 7 , 2020].

36 Schlapbach LJ, Aebischer M, Adams M, et al. Swiss neonatal network and follow-up group. impact of sepsis on neurodevelopmental outcome in a Swiss national cohort of extremely premature infants. Pediatrics 2011;128:e348-57.

37 Bayley N. Bayley scales of infant and toddler development: administration manual. San Antonia: Psychorp, 2006.

38 Kavas N, Arısoy AE, Bayhan A, et al. Neonatal sepsis and simple minor neurological dysfunction. Pediatr Int 2017;59:564-9.

39 Roid GH. Stanford Binet intelligence scales. Itasca: Riverside Pub, 2003

40 Wechsler D. Wechsler intelligence scale for children. San Antonia: PsychCorp, 2003.

41 Wechsler D. WPPSI : Wechsler Preschool and Primary Scale of Intelligence. New York: PsychCorp, 1967

42 The World Bank. World bank country and lending groups. [Internet] Available: https://datahelpdesk.worldbank.org/knowledgebase/ articles/906519-world-bank-country-and-lending-groups [Accessed 25 October 2019].

43 Higgins JP, Altman DG, Gøtzsche PC, et al. Cochrane bias methods group; Cochrane statistical methods group. The Cochrane collaboration's tool for assessing risk of bias in randomised trials. BMJ 2011;343:d5928.

44 Sterne J, Bradburn M, Egger M. Meta-Analysis in stata, in systematic reviews in health care: meta-analysis in context. $B M$ Publishing2008:347-69.

45 Shane AL, Sánchez PJ, Stoll BJ. Neonatal sepsis. Lancet 2017;390:1770-80.

46 Sucksdorff M, Lehtonen L, Chudal R, et al. Preterm birth and poor fetal growth as risk factors of Attention-Deficit/ hyperactivity disorder. Pediatrics 2015;136:e599-608.

47 Van Hus JW, Potharst ES, Jeukens-Visser M, et al. Motor impairment in very preterm-born children: links with other developmental deficits at 5 years of age. Dev Med Child Neurol 2014;56:587-94.

48 Lee E-J, Lee S-Y. The effects of early-stage neurodevelopmental treatment on the growth of premature infants in neonatal intensive care unit. J Exerc Rehabil 2018;14:523-9.

49 Ullenhag A, Persson K, Nyqvist KH. Motor performance in very preterm infants before and after implementation of the newborn individualized developmental care and assessment programme in a neonatal intensive care unit. Acta Paediatr 2009;98:947-52.

50 Vanderveen JA, Bassler D, Robertson CMT, et al. Early interventions involving parents to improve neurodevelopmental outcomes of premature infants: a meta-analysis. J Perinatol 2009;29:343-51.

51 Ohlsson A, Jacobs SE. NIDCAP: a systematic review and meta-analyses of randomized controlled trials. Pediatrics 2013;131:e881-93.

52 Hughes AJ, Redsell SA, Glazebrook C. Motor development interventions for preterm infants: a systematic review and metaanalysis. Pediatrics 2016;138:pii: e20160147.

53 Spittle A, Orton J, Anderson PJ, et al. Early developmental intervention programmes provided post hospital discharge to prevent motor and cognitive impairment in preterm infants. Cochrane Database Syst Rev 2015;11:CD005495.

54 Goldstein B, Giroir B, Randolph A. International consensus conference on pediatric sepsis. International pediatric sepsis consensus conference: definitions for sepsis and organ dysfunction in pediatrics. Pediatr Crit Care Med 2005;6:2-8. 